A brief historical perspective of the Wiener-Hopf technique

Lawrie, Jane B. and Abrahams, I. David 2007

MIMS EPrint: 2007.210

Manchester Institute for Mathematical Sciences

School of Mathematics

The University of Manchester

\footnotetext{
Reports available from: http://eprints.maths.manchester.ac.uk/

And by contacting: The MIMS Secretary

School of Mathematics

The University of Manchester

Manchester, M13 9PL, UK
} 


\title{
A brief historical perspective of the Wiener-Hopf technique
}

\author{
Jane B. Lawrie • I. David Abrahams
}

Received: 16 August 2007 / Accepted: 16 August 2007 / Published online: 17 October 2007

(C) Springer Science+Business Media B.V. 2007

\begin{abstract}
It is a little over 75 years since two of the most important mathematicians of the 20th century collaborated on finding the exact solution of a particular equation with semi-infinite convolution type integral operator. The elegance and analytical sophistication of the method, now called the Wiener-Hopf technique, impress all who use it. Its applicability to almost all branches of engineering, mathematical physics and applied mathematics is borne out by the many thousands of papers published on the subject since its conception. The Wiener-Hopf technique remains an extremely important tool for modern scientists, and the areas of application continue to broaden. This special issue of the Journal of Engineering Mathematics is dedicated to the work of Wiener and Hopf, and includes a number of articles which demonstrate the relevance of the technique to a representative range of model problems.
\end{abstract}

Keywords Eberhard Hopf · Norbert Wiener · Wiener filter · Wiener-Hopf technique

\section{Norbert Wiener and Eberhard Hopf}

In retrospect, it seems an unlikely collaboration: American born Norbert Wiener (1894-1964) and Austrian born Eberhard Hopf (1902-1983). The former was of European Jewish descent ${ }^{1}$ whilst the latter was educated in Berlin and, despite the prevailing political climate of 1930s Germany, chose to return from a position at the Massachusetts Institute of Technology (MIT) to take up a chair at the University of Leipzig in 1936. Individually, both men were powerful mathematicians who contributed to quite diverse areas of mathematics. To the general public Norbert Wiener is widely recognised as the founder of modern cybernetics. To mathematicians, however, he is primarily known for his highly innovative and fundamental work in what is now termed stochastic processes. His interest in randomness began in the early 1920s with studies of Brownian motion. This led him to harmonic analysis, Tauberian theorems and eventually to Paley-Wiener theory which was subsequently used to study problems involving more

${ }^{1}$ Wiener's mother was from a German family whilst his father, Leo Wiener, originated from Bialystock a town then in Tsarist Russia.

J. B. Lawrie $(\varangle)$

Department of Mathematical Sciences, Brunel University, Uxbridge UB8 3PH, UK

e-mail: jane.lawrie@brunel.ac.uk

I. D. Abrahams

School of Mathematics, University of Manchester, Oxford Road, Manchester M13 9PL, UK

e-mail: i.d.abrahams@manchester.ac.uk 
general stochastic processes. Eberhard Hopf, on the other hand, is known primarily for his work in ergodic theory and partial differential equations-his bifurcation theory is a particular tour de force that is still used repeatedly today as a central element of stability analysis and dynamical systems theory.

The two men differed greatly in personality. Wiener is widely acknowledged as having been absent-minded ${ }^{2}$ and his papers were hard to read: sometimes difficult results appeared with scarcely a proof and at other times he would present a lengthy proof of a triviality! It is also said that Wiener's lectures were difficult and often without structure. In contrast, Hopf was an excellent communicator: he had the ability to illuminate the most complex subjects and render them palatable to his colleagues and even to non-specialists.

\section{The Wiener-Hopf technique}

In 1930, having completed his Habilitation in Mathematical Astronomy at the University of Berlin, Hopf received a fellowship from the Rockefeller Foundation to study classical mechanics with Birkhoff (1884-1944) at Harvard College Observatory. A year later, and with the help of Norbert Wiener (who was already established at MIT), he joined the Department of Mathematics at the Massachusetts Institute of Technology on a temporary contract. The collaboration between Wiener and Hopf was initiated by their mutual interest in the differential equations governing the radiation equilibrium of stars. In Wiener's own words [2], "The various types of particle which form light and matter exist in a sort of balance with one another, which changes abruptly when we pass beyond the surface of the star. It is easy to set up the equations for this equilibrium, but it is not easy to find a general method for the solution of these equations." Their collaboration resulted in the famous paper [3] entitled "Über eine Klasse singulärer Integralgleichungen" in which they established the tool by which such equations could be solved. Thus, the Wiener-Hopf technique was first propounded as a means to solve, for $f(x)$, an integral equation of the form ${ }^{3}$

$\int_{0}^{\infty} k(x-y) f(y) \mathrm{d} y=g(x), \quad 0<x<\infty$,

where $k(x-y)$ is a known difference kernel and $g(x)$ is a specified function defined over the half-line $x>0$. For general readers of this special issue the salient points of the technique are very briefly outlined here; full details can be found in the textbook by Noble [4], more on which will be mentioned later. The method proceeds by extending the domain of, or continuing, the integral equation (1) to negative real values of $x$. Thus, write

$$
\int_{0}^{\infty} k(x-y) f(y) \mathrm{d} y= \begin{cases}g(x), & 0<x<\infty, \\ h(x), & -\infty<x<0,\end{cases}
$$

where $h(x)$ is unknown. Fourier transformation of (2) then yields the typical Wiener-Hopf functional equation

$G_{+}(\alpha)+H_{-}(\alpha)=F_{+}(\alpha) K(\alpha)$

in which $H_{-}(\alpha)$ and $F_{+}(\alpha)$ are half-range Fourier transforms (defined over $-\infty$ to 0 and 0 to $\infty$, respectively) of the unknowns $h(x)$ and $f(x)$. By contrast, the quantities $G_{+}(\alpha)$ (half-range Fourier transform of $g(x)$ ) and $K(\alpha)$ (full-range Fourier transform of $k(x))$ are known functions. The product form of the right-hand side of this equation is due to the fact that the original integral operator is of convolution type. The subscripts $+(-)$ indicate that the respective functions are analytic in upper(lower) half regions of the complex $\alpha$-plane which can be shown to overlap to form a strip in which all the functions are analytic. The Wiener-Hopf procedure hinges on finding a product factorization for the Fourier-transformed kernel, in the form

$$
K(\alpha)=K_{+}(\alpha) K_{-}(\alpha)
$$

\footnotetext{
${ }^{2}$ It is said that, after several years teaching at MIT, Wiener and his family moved to a larger house a few blocks away. Knowing that her husband was forgetful, Mrs Wiener wrote his new address on a piece of paper and gave it to him. However, when he was leaving his office at the end of the day, Wiener discovered that he had lost her directions and, of course, he could not remember where the new house was. So he drove to his old house instead (thinking that his wife would eventually come there to look for him). There he saw a young child and asked her, "Little girl, can you tell me where the Wieners moved?" "Yes, Daddy," she replied, "Mommy said you'd probably be here, so she sent me to show you the way home." This and several other amusing anecdotes can be found in Ref. [1].

3 Shown here for simplicity as a first-kind integral equation, but it often occurs of second-kind.
} 
which enables (3) to be re-written as

$\frac{G_{+}(\alpha)}{K_{-}(\alpha)}+\frac{H_{-}(\alpha)}{K_{-}(\alpha)}=F_{+}(\alpha) K_{+}(\alpha)$.

Note that the factors on the right-hand side of (4) are also zero-free in their indicated half-planes of analyticity. Thus, on defining a sum-factorization ${ }^{4}$ of the form

$\frac{G_{+}(\alpha)}{K_{-}(\alpha)}=L_{+}(\alpha)+L_{-}(\alpha)$,

it follows that the equation may be re-expressed as

$L_{-}(\alpha)+\frac{H_{-}(\alpha)}{K_{-}(\alpha)}=F_{+}(\alpha) K_{+}(\alpha)-L_{+}(\alpha)$,

where the left-hand side is analytic in the lower half-plane and the right-hand side is analytic in the overlapping upper half region. Arguments involving analytic continuation now enable both sides of this equation to be equated to an entire function, $E(\alpha)$, say. Physical constraints on the behaviour of $f(x), g(x)$ and $k(x)$ as $x \rightarrow 0$, and correspondingly to their Fourier-transformed quantities in (7) as $|\alpha| \rightarrow \infty$, allows $E(\alpha)$ to be specified, and hence $H_{-}(\alpha)$ and $F_{+}(\alpha)$, are uniquely determined. Fourier inversion finally yields the unknown function $f(x)$.

\section{Developments and applications of the technique}

Interestingly, in their book, Carrier, Krook and Pearson [5] suggest that, in fact, Carleman (1892-1949) may have first developed the method in as early as $1921 .^{5}$ The authors, however, have been unable to substantiate this claim. In 1934 Hopf published the book "Mathematical problems of radiative equilibrium" [7] which provides a lucid account of the Wiener-Hopf technique in the context of the class of problems that first inspired the collaboration. It was at the end of his contract with MIT that Hopf took up a full professorship at the University of Leipzig. On the matter of Hopf's return to Germany, Wiener was uncritical. He knew that, particularly when set against the United State's economic depression, the post offered to Hopf was both lucrative and offered social prestige beyond that then available at MIT. He acknowledged that Hopf's views were not strongly pro-Nazi and felt that the position was better filled by a man of moderate views. Wiener feared, however, that Hopf's acceptance would severely damage his standing in the academic community. Indeed that seems to have been the case: in the years following the end of the second World War, Hopf suffered a substantial decrease in popularity which led to the neglect of his work and even to it being attributed to other mathematicians. It is, for example, suggested [8] that Hopf's name was dropped from the discrete version of the Wiener-Hopf equation, which is now referred to as the 'Wiener filter'.

During the 1940s it was discovered that problems involving diffraction by semi-infinite planes/geometries could, by using Green's theorem, be formulated in terms of integral equations of the form of (1). The integral equation could then be solved, as described above, by applying Fourier Transforms and using the Wiener-Hopf technique. Although, somewhat cumbersome, the method provided a direct route for solving problems such as the famous Sommerfeld half-plane problem [9], and practical applications of and extensions to the technique began to appear frequently in the literature (see, for example, the now classic papers by Copson [10], Carlson and Heins [11, 12] and Levine and Schwinger [13]). It was not, however, until Jones [14] demonstrated that the method could be considerably simplified by applying transforms directly to the boundary-value problem that the versatility and power of the method began to be realised. Jones' method by-passes the initial derivation of the semi-infinite integral equation of the form (1) and yields directly the complex Wiener-Hopf functional equation (3).

\footnotetext{
${ }^{4}$ It will be seen in the forthcoming articles in this Special Issue that sum and product factorizations of many scalar functions are obtained in a straightforward manner by use of Cauchy's integral formula.

5 Note that any Wiener-Hopf problem can be recast into a special class of Riemann-Hilbert problem defined on an infinite line. The standard solution procedure is analogous to that discussed above, but there are a few differences including the application of Plemelj formulae for the factorization step (see e.g. Muskhelivshvili's classic book [6]). To the authors' knowledge, the development of solution methods for relevant Riemann-Hilbert problems do not pre-date Wiener and Hopf's work.
} 
By the mid-1950s the method was well established and Ian N. Sneddon (1919-2000), a seminal figure in Scottish applied mathematics [15] and solid mechanics in particular, realised the need for a research monograph on the technique. Interestingly Sneddon, who was internationally recognised for his work on transform methods for the solution of partial differential equations and the author of several outstanding textbooks on this subject, also offered a major contribution to the field of mathematics through his work editing translations of major Russian texts - many for the successful series "International series of monographs in pure and applied mathematics". It was in his capacity as editor of this series that he suggested to Ben Noble (1922-2006) that he might like to write a research monograph on the technique, and the concept of this book may well have been conceived during the period 1952-1955 when both men worked at the University of Keele in the UK. It was not until 1958, however, (by which time they were both in Glasgow) that the now classic textbook "The Wiener-Hopf technique"6 appeared. This book has enduring popularity amongst all those who rank the Wiener-Hopf technique as a potent tool of the trade. Indeed, one could almost say that it has become the 'Bible' of the Wiener-Hopf practitioner! The presentation of Noble's book may lack the polish of many classic texts; this, however, does not detract from its appeal, which is two-fold. Not only does this book deal with the application of the Wiener-Hopf technique to problems involving semi-infinite geometries, and comprehensively discuss a wide range of extensions to, limitations of and approximations to the method, but on almost every page can be found an interesting and relevant 'tit-bit' - neat factorizations are tucked away alongside valuable references and a wealth of interesting ideas.

\section{Extensions, variations and applications of the technique}

Of course, during the 50 years that have elapsed since the first edition of Noble's book, the method has advanced on almost every front. At this point is is worthwhile remarking that the method is ideally suited to solve two-part twoor three-dimensional boundary-value problems involving a governing equation (such as Laplace's or Helmholtz') with mixed boundary conditions along one infinite coordinate line. ${ }^{7}$ That is, for a two-dimensional problem, one condition for say, $x<0, y=0$ and a different condition for $x>0, y=0$. It is natural to ask what happens to the Wiener-Hopf formulation for three seemingly simple variations (henceforth referred to as type I-III) of this geometry. It is fair to say that curiosity about these variations has inspired and driven many of the recent developments in Wiener-Hopf theory.

Type I geometry arises if the mixed conditions are imposed for $x<0$ and $x>0$ but at different values of $y$. For many such problems this results in coupled Wiener-Hopf equations that can be expressed in matrix form. Matrix Wiener-Hopf equations arise from a huge variety of models in a multitude of areas, including mathematical physics, fluid and solid mechanics, wave diffraction and even mathematical finance. This class represents one of the biggest growth areas in the study and development of the Wiener-Hopf technique. Matrix Wiener-Hopf kernels are fundamentally distinct from their scalar counterparts in that there is no algorithmic approach to determining the factorization (4) of the transformed kernel [16]. Exact factorization can be achieved for matrices with certain special features: those that are upper (or lower) triangular in form; those that are of Khrapkov-Daniele, i.e., commutative, form (see [17-20]); those whose elements comprise meromorphic functions [21,22]; kernels with special singularity structure that allows the Wiener-Hopf equation to be recast into uncoupled Riemann-Hilbert problems [23-25]; and $N \times N$ matrices with special algebraic or group structure [26-28]. For more details on exact matrix kernel factorization the interested reader is referred to the last mentioned article and to references cited in [29].

In general, however, this class of problems is, as yet, intractable to exact techniques. For this reason a number of approximate methods of kernel factorization have been developed since the 1950s. Most schemes address scalar factorization using a variety of direct ad-hoc and rational approximate approaches [30-33], including a novel use of the method of matched asymptotic expansions [34] to simplify the kernel. For matrix kernels containing exponential

\footnotetext{
6 The full title of Noble's book is actually "Methods based on the Wiener-Hopf technique for the solution of partial differential equations".

7 This coordinate may be temporal as well as spatial.
} 
phase-type factors an integral equation approach may be used to solve the system of Wiener-Hopf equations (see e.g. [35]). Abrahams has successfully employed Padé approximants to obtain explicit exact factorizations of both approximate scalar [36] and matrix kernels. The latter approach has allowed a number of long-outstanding problems to be solved recently $[29,37,38]$.

A type II geometry arises if one confines the boundary data to be specified along $y=0$ but allows a three-part condition, for example, different conditions for each of $x<-a, x<|a|$ and $x>a$. In this case, a modified Wiener-Hopf equation is obtained which is distinct from the usual formulation in that it contains three unknown functions (each analytic in specified regions of the complex plane) and cannot be solved exactly using the usual analytic continuation arguments. The solution to such problems can, however, be cast as a pair of coupled integral equations which may be solved approximately in certain limiting cases [4,39]. It is not untypical for type I and type II variations in geometry to occur together; in such cases a modified matrix W-H equation results which can usually only be solved by approximate methods (see e.g. [40]). It is worthwhile noting that for three-part problems involving propagation in closed waveguides, a mode-matching approach offers a convenient alternative; see e.g. [41]. This method enables problems that combine both type I and type II features to be reduced to an infinite system of linear algebraic equations that can be numerically solved by truncation. It is, however, inapplicable to problems of infinite extent in the direction orthogonal to the waveguide axis, i.e., when there are radiation terms in the far field.

Type III geometry arises when the abrupt change of boundary condition is no longer confined to the 'in-plane' situation. Thus, for example, one condition may hold for $x<0, y=0$ and the other for $x=0, y>0$ producing a corner (or wedge) which, in this case, is of interior angle $\pi / 2$ but could be of arbitrary angle. The seminal articles to address the scattering of incident waves in a wedge with non-simple boundary conditions were by Maliuzhinets [42], and Williams [43]. Working independently, they both considered a wedge with impedance boundary conditions and used a Sommerfeld integral representation of the diffracted field to reduce the boundary-value problem to a pair of functional difference equations of the form

$\{\sin X-\sin s\} f(s \pm \beta)-\{\sin s+\sin X\} f(-s \pm \beta)=0$,

where $4 \beta$ is the wedge angle and $X$ is a constant which is related to the physical properties of the wedge boundaries. Maliuzhinets solved these by introducing a new special function (now referred to as the Maliuzhinets function) whilst Williams used the Barnes double-gamma function. ${ }^{8}$ The solution method involves deriving a function $f(s)$ that both satisfies (8) and, reminiscent of the Wiener-Hopf technique, has no singularities in a vertical strip in the complex $s$-plane.

The relationship between the functional difference-equation method of solution for wedge problems, and the Wiener-Hopf technique is easily shown for a wedge of angle $\pi$ (in which case $\beta=\pi / 4$ in (8)). Indeed, Abrahams and Lawrie [44] used this relationship to recast the problem of factorizing a particular class of Wiener-Hopf kernels by formulating a difference equation representation for the factors. For arbitrary angle the relationship between the two methods is less obvious. In recent papers, however, Daniele $[45,46]$ has demonstrated the direct equivalence between the method of Maliuzhinets for wave scattering in a wedge with impedance barriers [42] and Khrapkov-Daniele commutative matrix kernel factorization.

Maliuzhinets' work has been advanced in a number of directions by various Russian scientists in recent years; see, for example, the excellent review by Osipov and Norris [47] and works by Budaev [48], Bernard [49], Osipov [50], Lyalinov and Zhu [51]. In particular, the development of the functional difference-equation method for solving problems in a wedge-shaped domain with boundaries that are described by high-order conditions (such as those modelling a membrane or elastic plate) is a significant, and non-trivial extension [52-54]. Building upon these works, mathematicians have more recently focused their attention on three-dimensional scattering problems involving conical geometries [55-59]. It is an interesting and open challenge to understand the relationship between the methods developed for the conical problems and standard Wiener-Hopf analysis.

\footnotetext{
${ }^{8}$ Since the Maliuzhinets functions may be expressed in terms of the Barnes double-gamma functions the methods of Maliuzhinets and Williams are essentially equivalent.
} 


\section{Wiener-Hopf special issue}

As mentioned earlier, an enormous variety of physically important problems can be cast into equations of Wiener-Hopf form, and all have the characteristic feature of distinct boundary conditions defined on disjoint lines or line segments. Depending on the given problem, such boundary conditions may be defined in space or time, and may range over discrete or continuous independent variables. The Wiener-Hopf technique has found application in an very wide variety of research areas, including the diffraction of acoustic, elastic and electromagnetic waves, crystal growth [60], fracture mechanics, flow problems [61], diffusion models [62], geophysical applications [63] and mathematical finance. A simple search of the science literature will reveal the many thousands of articles that have employed Wiener and Hopf's elegant method.

This Special Issue of the Journal of Engineering Mathematics is presented to mark the 75 years since the Wiener-Hopf technique was first published [3]. The articles contained herein are not chosen to indicate the current state-of-the-art regarding advanced Wiener-Hopf methods, for example on matrix Wiener-Hopf factorization, as this would be neither useful nor particularly interesting for a general readership. Also, the Issue does not aim to offer a complete collection of current areas where the Wiener-Hopf technique has found application; this would demand several volumes, rather than a single issue! Instead, the following collection of articles serves to present the readership of the Journal of Engineering Mathematics with a small sample of cutting-edge works in the field-it offers a set of interesting applications of the Wiener-Hopf technique, together with a couple of papers demonstrating how the technique can and has been extended.

The article by Antipov and Willis discusses constant crack growth in a viscoelastic material, with emphasis on the field around the crack tip. Solid mechanics in general, and fracture mechanics in particular, is a subject in which the Wiener-Hopf technique has proved an immensely useful tool. This is because the field quantities are strongly dominated by points of rapid or instantaneous change in boundary conditions, in the vicinity of which Wiener-Hopf or related techniques are ideally suited. This point of view is reinforced in the article by Norris and Abrahams in which crack-growth is again studied. In this case the crack growth model is somewhat simpler but the solution method employs matched asymptotic expansions as well as the Wiener-Hopf technique in order to examine the stability of running waves along the propagating crack tip.

Diffraction theory is another area in which the field variables are dominated by points where the geometry or boundary conditions change abruptly, and this may perhaps be the reason why it has found the most application for the Wiener-Hopf technique. It is natural therefore that papers are included on this topic. Rawlins considers a model of reflection and transmission of acoustic waves in a bifurcated, impedance-lined waveguide - a problem which leads to a standard scalar Wiener-Hopf equation. In contrast, Lyalinov and Zhu examine wave scattering by a semi-transparent impedance cone. The boundary-value problem is recast as a second-order functional difference equation ${ }^{9}$ which class, as mentioned earlier, was shown by Daniele [45] bear a close relationship with matrix Wiener-Hopf systems. Here, however, the authors choose an alternative evaluation approach, employing the numerical solution of a Fredholm integral equation.

The article by Green et al. addresses an important problem in mathematical finance, namely the pricing of discretely monitored barrier options. For simplicity, the authors investigate European options in a Gaussian BlackScholes formulation. Via Fourier and $z$-transforms the problem is reduced to Wiener-Hopf form, and an exact solution is obtained. This article is indicative of the substantial interest in the Wiener-Hopf technique in this new and burgeoning area of applied mathematics.

Aero-acoustics is another topic in which researchers have made excellent use of the Wiener-Hopf technique over some three or more decades. Computational methods to extract the very small sound field from a dominant background flow field are fraught with difficulties, and so analytical methods have proved extremely useful. Rienstra, a senior practitioner in this area, presents the 2007 annual Lighthill paper of the Journal of Engineering Mathematics. In it he discusses sound scattering in a flow over the interface between hard and soft impedance cylindrical boundaries. Of particular interest is the predicted onset of instability in the model.

\footnotetext{
${ }^{9}$ Equivalent to a Carleman boundary-value problem for analytic vectors.
} 
Finally, the article by Shanin and Doubravsky discusses a new approach which can be seen as either a generalisation of the Wiener-Hopf technique or as an alternative method. The scheme involves, for problems of a particular class, extending 'physical space' so that it is multi-sheeted. By this means a field solution may be written down which, when restricted to the principal sheet, satisfies the necessary jump conditions at the boundaries.

To conclude, it is interesting to note, on flicking through some of the many biographies of Wiener and Hopf (e.g. [64]), that few authors give the Wiener-Hopf technique the prominence that it deserves, preferring to focus on other aspects of the works of these two great men. Yet, the Wiener-Hopf technique has enabled the solution of numerous timely and physically relevant problems and, furthermore, has acted as a catalyst for the generation of many sophisticated extensions and variations of the method. Wiener and Hopf would surely be pleased to know that, 75 years after its conception, the Wiener-Hopf technique remains a source of inspiration to mathematicians, physicists and engineers working in many diverse fields. To quote Wiener "Mathematics is too arduous and uninviting a field to appeal to those to whom it does not give great rewards." Thankfully, he and Hopf developed a tool by which less prodigious scientists are able to reap a few such rewards!

\section{References}

1. http://www.en.wikipedia.org/wiki/Norbert_Wiener (Accessed on 24 September 2007)

2. Wiener N (1956) I am a Mathematician. Doubleday \& Co., Inc., Garden City

3. Wiener N, Hopf E (1931) Über eine klasse singulärer integralgleichungen. Sem-Ber Preuss Akad Wiss 31:696-706

4. Noble B (1988) Methods based on the Wiener-Hopf Technique, 2nd edn. Chelsea Publishing Company, New York

5. Carrier GF, Krook M, Pearson CE (2005) Functions of a complex variable. SIAM Publishing, Philadelphia

6. Muskhelishvili NI (1953) Singular integral equations, Translated from second edition Moscow (1946) by J.R.M. Radok. Noordhoff, Groningen

7. Hopf E (1934) Mathematical problems of radiative equilibrium. Cambridge Tract 31, Cambridge University Press, Cambridge

8. http://www.history.mcs.st-andrews.ac.uk/Biographies/Hopf_Eberhard.html (Accessed on 24 September 2007)

9. Sommerfeld A (1896) Mathematische theorie der diffraction. Math Ann 47:317-374

10. Copson ET (1946) On an integral equation arising in the theory of diffraction. Quart J Math 17:19-34

11. Carlson JF, Heins AE (1947) The reflection of an electromagnetic plane wave by an infinite set of plates, I. Quart Appl Math 4:313-329

12. Carlson JF, Heins AE (1947) The reflection of an electromagnetic plane wave by an infinite set of plates, II. Quart Appl Math 5:82-88

13. Levine H, Schwinger J (1948) On the radiation of sound from an unflanged circular pipe. Phys Rev 73:383-406

14. Jones DS (1952) A simplifying technique in the solution of a class of diffraction problems. Quart J Math 3:189-196

15. http://www.history.mcs.st-andrews.ac.uk/Biographies/Sneddon.html (Accessed on 24 September 2007)

16. Heins AE (1950) Systems of Wiener-Hopf equations. In: Proceedings of Symposia in Applied Mathematics II. McGraw-Hill, pp 76-81

17. Khrapkov AA (1971) Certain cases of the elastic equilibrium of an infinite wedge with a non-symmetric notch at the vertex, subjected to concentrated forces. Appl Math Mech (PMM) 35:625-637

18. Khrapkov AA (1971) Closed form solutions of problems on the elastic equilibrium of an infinite wedge with nonsymmetric notch at the apex. Appl Math Mech (PMM) 35:1009-1016

19. Daniele VG (1978) On the factorization of Wiener-Hopf matrices in problems solvable with Hurd's method. IEEE Trans Antennas Propagat 26:614-616

20. Rawlins AD (1975) The solution of a mixed boundary value problem in the theory of diffraction by a semi-infinite plane. Proc Roy Soc London A 346:469-484

21. Rawlins AD (1985) A note on Wiener-Hopf matrix factorization. Quart J Mech Appl Math 38:433-437

22. Idemen M (1979) A new method to obtain exact solutions of vector Wiener-Hopf equations. Zeit Angew Math Mech 59:656-658

23. Hurd RA (1976) The Wiener-Hopf Hilbert method for diffraction problems. Canad J Phys 54:775-780

24. Rawlins AD, Williams WE (1981) Matrix Wiener-Hopf factorization. Quart J Mech Appl Math 34:1-8

25. Williams WE (1984) Recognition of some readily "Wiener-Hopf” factorizable matrices. IMA J Appl Math 32:367-378

26. Jones DS (1984) Commutative Wiener-Hopf factorization of a matrix. Proc Roy Soc Lond A 393:185-192

27. Jones DS (1984) Factorization of a Wiener-Hopf matrix. IMA J Appl Math 32:211-220

28. Veitch BH, Abrahams ID (2007) On the commutative factorization of $n \times n$ matrix Wiener-Hopf kernels with distinct eigenvalues. Proc Roy Soc London A 463:613-639

29. Abrahams ID (1997) On the solution of Wiener-Hopf problems involving noncommutative matrix kernel decompositions. SIAM J Appl Math 57:541-567 
30. Koiter WT (1954) Approximate solution of Wiener-Hopf type integral equations with applications, parts I-III. Koninkl Ned Akad Wetenschap Proc B 57:558-579

31. Carrier GF (1959) Useful approximations in Wiener-Hopf problems. J Appl Phys 30:1769-1774

32. Kranzer HC, Radlow J (1962) Asymptotic factorization for perturbed Wiener-Hopf problems. J Math Anal Appl 4:240-256

33. Kranzer HC, Radlow J (1965) An asymptotic method for solving perturbed Wiener-Hopf problems. J Math Mech 14:41-59

34. Crighton DG (2001) Asymptotic factorization of Wiener-Hopf kernels. Wave Motion 33:51-65

35. Abrahams ID, Wickham GR (1990) General Wiener-Hopf factorization of matrix kernels with exponential phase factors. SIAM J Appl Math 50:819-838

36. Abrahams ID (2000) The application of Padé approximants to Wiener-Hopf factorization. IMA J Appl Math 65:257-281

37. Abrahams ID (1996) Radiation and scattering of waves on an elastic half-space; a noncommutative matrix Wiener-Hopf problem. J Mech Phys Solids 44:2125-2154

38. Abrahams ID (2002) On the application of the Wiener-Hopf technique to problems in dynamic elasticity. Wave Motion 36:311-333

39. Jones DS (1952) Diffraction by a waveguide of finite length. Proc Camb Phil Soc 48:118-134

40. Owen GW, Abrahams ID (2006) Elastic wave radiation from a high frequency finite-length transducer. J Sound Vib 298:108-131

41. Lawrie JB, Guled IMM (2006) On tuning a reactive silencer by vaying the position of an internal membrane. J Acoust Soc Amer 120:780-790

42. Maliuzhinets GD (1958) Excitation, reflection and emission of surface waves from a wedge with given face impedances. Soviet Phys Doklady 3:752-755

43. Williams WE (1959) Diffraction of an E-polarized plane wave by an imperfectly conducting wedge. Proc R Soc Lond A 252:376393

44. Abrahams ID, Lawrie JB (1995) On the factorization of a class of Wiener-Hopf kernels. IMA J Appl Math 55:35-47

45. Daniele VG (2003) The Wiener-Hopf technique for impenetrable wedges having arbitrary aperture angle. SIAM J Appl Math 63:1442-1460

46. Daniele VG, Lombardi G (2006) Wiener-Hopf Solution for impenetrable wedges at skew incidence. IEEE Trans Antenn Prop 54:2472-2485

47. Osipov AV, Norris AN (1999) The Malyuzhinets theory for scattering from wedge boundaries: a review. Wave Motion 29:313-340

48. Budaev B (1995) Diffraction by wedges. Longman Scientific, London

49. Bernard JML (1998) Diffraction at skew incidence by an anisotropic impedance wedge in electromagnetism theory; a new class of canonical cases. J Phys A: Math Gen 31:595-613

50. Osipov AV (2004) A hybrid technique for the analysis of scattering by impedance wedges. In: Proceedings of URSI Int Symp Electromagn Theory Pisa, Vol. 2, pp. 1140-1142

51. Lyalinov MA, Zhu NY (2003) Exact solution to diffraction problem by wedges with a class of anisotropic impedance faces: oblique incidence of a plane electromagnetic wave. IEEE Trans Antennas Propag 51:1216-1220

52. Abrahams ID, Lawrie JB (1995) Travelling waves on a membrane: reflection and transmission at a corner of arbitrary angle, I. Proc R Soc London A 451:657-683

53. Osipov AV (1996) Diffraction by a wedge with higher-order boundary conditions. Radio Sci 31:1705-1720

54. Norris AN, Osipov AV (1997) Structural and acoustical wave interaction at a wedge-shaped junction of fluid-loaded plates. J Acoust Soc Amer 101:867-876

55. Smyshlyaev VP (1993) The high-frequency diffraction of electromagnetic waves by cones of arbitrary cross sections. SIAM J Appl Math 53:670-688

56. Babich VM, Dement'ev DB, Samokish BA, Smyshlyaev VP (2000) On evaluation of the diffraction coefficients for arbitrary 'nonsingular' directions of a smooth convex cone. SIAM J Appl Math 60:536-573

57. Jones DS (1997) Scattering by a cone. Q J1 Mech Appl Math 50:499-523

58. Bernard JML, Lyalinov MA (2001) Spectral domain solution and asymptotics for the diffraction by an impedance cone. IEEE Trans Antennas Propagat 49:1633-1637

59. Antipov YA (2002) Diffraction of a plane wave by a circular cone with an impedance boundary condition. SIAM J Appl Math 62:1122-1152

60. Kuiken HK (1985) Edge effects in crystal growth under intermediate diffusive-kinetic control. IMA J Appl Math 35:117-129

61. Boersma J (1978) Note on an integral equation of viscous flow theory. J Eng Math 12:237-243

62. Boersma J, Indenkleef JJE, Kuiken HK (1984) A diffusion problem in semconductor technology. J Eng Math 18:315-333

63. Mysak LA, LeBlond PH (1972) The scattering of Rossby waves by a semi-infinite barrier. J Phys Oceanogr 2:108-114

64. Levinson N (1966) Norbert Wiener. Bull Amer Math Soc 72:1-32 